\title{
Information Visualization Techniques for Exploring Oil Well Trajectories in Reservoir Models
}

\author{
Sowmya Somanath* \\ Sheelagh Carpendale ${ }^{\dagger}$ \\ Ehud Sharlin \\ Mario Costa Sousa ${ }^{\S}$ \\ Department of Computer Science \\ University of Calgary, Calgary, Canada
}

\begin{abstract}
We present a set of interactive 3D visualizations, designed to explore oil/gas reservoir simulation post-processing models. With these visualizations we aim to provide reservoir engineers with better access to the data within their 3D models. We provide techniques for exploring existing oil well trajectories, and for planning future wells, to assist in decision making. Our approach focuses on designing visualization techniques that present the necessary details using concepts from information visualization. We created three new visualization variations - lollipop-up, information circles and path indicator, which present well trajectory specific information in different visual formats. Our paper describes these visualizations and discusses them in context of our exploratory evaluation.
\end{abstract}

Keywords: 3D Reservoir Visualization, Reservoir Engineering, Information Visualization.

Index Terms: H.5.m [Information Interfaces and Presentation (e.g., HCI)]: Miscellaneous-; J.2 [Physical Sciences and Engineering]: Earth and Atmospheric Sciences-

\section{INTRODUCTION}

Oil and gas reservoirs are sub-surface portions of the earth which contain hydrocarbons. The fundamental goal of reservoir engineering studies is to assess and evaluate these entities and to design optimum ways to extract oil and gas. However, reservoir data acquisition is costly and therefore, engineers have only access to limited spatio-temporal information about the reservoirs [8]. The majority of available information comes from sensor measurements (e.g. seismic geophones, pressure transducers) and experimental results of rock samples (e.g. core measurements). These are combined with multi-disciplinary experts advice to develop a realistic 3D geological model [8]. However, complexity, size and multi-resolution data are amongst many challenges for proper data integration and efficient analysis. These require advanced visualization tools to better understand and access the data $[9,23]$.

In practice, the oil and gas exploration and production cycle (E\&P) consist of several stages of data gathering, simulation and data analysis. Of those many stages, our work is situated at one of the last phases of the exploration cycle, called the post-processing stage. A post-processing stage of reservoir studies includes simulations of different scenarios of the reservoir models for history matching, prediction and forecasting [18]. At the history matching stage the reservoir engineer needs to manipulate some of the uncertain reservoir properties and run multiple reservoir simulations in order to provide a better match with the real production data.

\footnotetext{
*e-mail:ssomanat@ucalgary.ca

$\dagger$ e-mail:sheelagh@ucalgary.ca

†e-mail:ehud@ucalgary.ca

§e-mail:smcosta@ucalgary.ca

Graphics Interface Conference 2014

7-9 May, Montreal, Quebec, Canada

Copyright held by authors. Permission granted to

CHCCS/SCDHM to publish in print form, and

ACM to publish electronically.
}

This will add a further complexity as the size of the data increases rapidly. The simulation data are time varying and multi-attribute in nature, requiring techniques that support adequate exploration of those many parameters.

At the post-processing stage, a 3D cellular model, an approximation of the real reservoir, is used for exploring the various parameter configurations and fluid flows. The structure of this 3D reservoir model is usually represented by irregular corner point 3D grid [17] (irregular hexahedron geometries) consisting of thousands to millions of cells, each of which is associated with many static and dynamic properties (e.g. porosity, permeability, pressure, saturation and etc.). Apart from the cell properties, the simulation models also contain information about existing well trajectories within the reservoir (i.e. collections of perforated cells through which a 3D line passes). Thereby, the complexity of the models arising from multiple cells attributes and the 3D geometry motivates the need to invent various visual exploration techniques in order to help reservoir engineers to gain a clear picture of the relationship between these data attributes.

Among the various tasks conducted at the post-processing stage, we focus on the exploration of well trajectories. Exploring well trajectories is important as it helps in future decision making for oil well placements, a cost-intensive activity, requiring detailed analysis. In particular, we focus on a small subset of visualization tasks: learning about the existing well trajectories in a reservoir post-processing cellular model, and exploration of favorable locations for new trajectories.

During the post-processing stage, reservoir engineers analyze well performance primarily through looking at pressure curves and production rates. Reservoir production is often formulated as an optimization problem. Visual analysis with new spatial reservoir visualization tools can complement standard well production visualizations; visual tools can help develop a better understanding of the relationship between the attributes, the individual cells and the model structure as a whole. To this end, we present the design and implementation of three new information visualization techniques - lollipop-up visualization, information circles and path indicator, which present well trajectory specific information in different styles.

The fundamental contribution of our work is providing the subdomain of reservoir engineering post- processing studies with a collection of information visualization techniques that can be used for the exploration of well trajectories. Each of the visualizations was evaluated in a preliminary evaluation session with six participants and the results are presented followed by a discussion section. The big picture encompassing these efforts is to create a system wherein the engineer can study existing wells and use the learned knowledge to better predict where new wells can be placed. However, in this initial research we approach these bigger concepts by focusing on learning more from the engineers about their expectations in regards to well exploration and new well creation systems, leading to better design applications for such use cases. 


\section{Reservoir Model}

Reservoir post-processing models are the end result of multiple simulation scenarios. The model employed in our experiment consists of the following four types of information: (a) structural information, (b) time steps, (c) cell specific information (geological property values - both static and dynamic) and (d) well trajectory specific information (type of well, length of well, perforation cells etc.). The structure of the 3D model consists of corner point cells [17], irregular hexagonal geometries, arranged along three dimensions $(\mathrm{i}, \mathrm{j}, \mathrm{k})$. The $\mathrm{i}$ and $\mathrm{j}$ dimensions correspond to the cells in the $\mathrm{x}$ and $\mathrm{y}$ directions of the $3 \mathrm{D}$ space and $\mathrm{k}$ dimension corresponds to the layers of the 3D model. For example, the model used in our experiment (Figure 1a) consists of 33000 cells, i.e. $39 \times 42 \times 20$ cells in the $\mathrm{i}, \mathrm{j}$ and $\mathrm{k}$ directions respectively. The arrangement of the cells represents both spatial continuity as well as discontinuities to accommodate geological structures such as faults. The model used in this experiment consists of four dynamic properties and ten static properties. Apart from geological properties, the models also encompass well specific information. Each of the well trajectories are represented as a collection of perforated cells, and the centroids of these cells are an approximation to the 3D points of the well trajectory within the simulation grid.

\subsection{Visual Mapping}

To visualize geological properties, reservoir models are mapped to different color maps corresponding to the range of the property value. This mapping is usually limited to a single geological property. However, the effect of one property over another is of interest to a reservoir engineer, especially in the context of studying well trajectories [21]. Thus, to better support well trajectory exploration tasks a proof of concept protoype was developed wherein we group two geological properties (oil pressure and rock porosity) to create a color map that indicates their combined effects. Pressure and porosity were chosen as the two geological properties because in the most simple case, rock should be porous and pressure should be high in order for oil to flow. To formalize a term for regions of low pressure and low porosity (blue regions of the model in Figure 1a), we call them unfit regions, corresponding to an engineering interpretation of them being unsuitable for placement of well trajectories. Similarly, regions of high pressure and high porosity (green regions of the model in Figure 1a) correspond to regions of better fit. These types of combination values can be calculated based on many different correlation equations and ratios as per the experts discretion [16]. Depending on the calculation method employed, the resulting color pattern for the model can be different from the one shown in Figure 1. In our prototype the combined value calculation is simple and is defined as a percentage of the number of unfit cells per spatial partition (uniform spatial division) to the number of total cells per partition. Unfit cells here correspond to those cells whose porosity and pressure value lie within a threshold identified by trial and error using the given reservoir data values.

\section{Related Work}

Data clarification is one of the major goals of visualizations in general. However, the pertinent information could either be in portions of the context or it could be correlations resulting from the analysis of the data. In particular 'focus and context' is one family of visualization techniques which can help the viewer to learn about an area of interest (pertinent parts of the whole data set) while retaining the rest of the context in intelligent ways to avoid visual clutter and occlusion in $3 \mathrm{D}$ volumetric data $[4,5,6,8,10,24]$. Several interesting variations have been suggested for performing focus and context by using styles such as fisheye views [11], distortion [5, 19], magnification [25] and temporary displacement of context [21]. In the realm of volumetric data, concepts such as importance driven rendering [24] and illustrative rendering techniques
[4] have been suggested. Sultanum et al. [21] implemented a focus and context technique to visualize well trajectories by creating a "V" shaped opening in the region of the selected wells. Depending on the task, removing parts of the context (of the reservoir model) was suggested as not acceptable by engineers. For example, in the case of tasks such as trend analysis, the engineer is interested not just in the important entity (well trajectory), but also the entity in relation with the other parts of the context [21].

Motivated by this domain requirement, one of our visualizations (lollipop-up visualization, Figure 1) was influenced by techniques such as those proposed by Ware and Lewis [25] and Taerum et al.[22], which help to retain the context (unlike the distortion and cut-away techniques) and extract the important information to visualize them outside the context. DragMag [25] is a technique wherein the concept of offsets was used. The area of importance is displaced from the context, magnified and connected to the actual context using vertical lines for better viewing. Taerum et al. [22] used the concept of a contextual close-up to view the areas of importance. The difference between our lollipop-up visualization and these techniques is that we displace the actual 3D well blocks (i.e. no magnification) above the reservoir surface to help the viewer learn about the internal blocks. This way we are able to maintain the geometric information, continuities and discontinuities, and the actual scale of the geometric model, thus showing how the well is actually drilled underground. In the realm of reservoir engineering, offsets have been used by Lee et al. [15] in a manner similar to the bubble maps $[1,2,13]$, which visualize cumulative statistical information (e.g. water injection rate over two years) using a single cube or sphere at the end of a stick for each well in the model. Lollipop-ups on the other hand, provide information about each individual perforated cell, rather than a cumulative result. Hence, they can provide insights about the underground trajectory cells both in terms of geometry and property values. Similar to offsets, the concept of lenses or 3D probes applied to seismic data survey was proposed by Castanie et al. [7].

In multi-dimensional datasets, apart from the need to focus on areas of importance, correlation between multiple instances of similar entities is also important. For example, it is important to learn about the similarities in the geological property distribution of the perforated cells for two or more trajectories. In other words, the similarities or differences between two or more well trajectories are of interest. There have been several techniques employed to visualize correlations. Some of the current research in this area includes: the volume reformation visualization technique explained by Lampe et al. to compare well trajectories [14], and the focus and context technique by Gasteiger et al. involving the use of lenses to better support correlation [12].

In our approach, we return to using visualization variables [3] to support correlation exploration. We present information circles and the path indicator visualization, which present pre-processed information allowing engineers to gain access to statistical information when needed. Our approach is to augment the 3D grid visualization of the reservoir and its contextual geology with information visualization additions representing more abstract aspects of the data using factors such as color, shape and size [3]. These visualization augmentations provide more information about single well trajectories, multiple well trajectories and individual cells of the model.

\section{Visualizations}

In this section we describe the three visualization variants: lollipopup, information circles and path indicator. All the visualizations have been implemented for the Microsoft Surface 1.0 using C\# and XNA. 


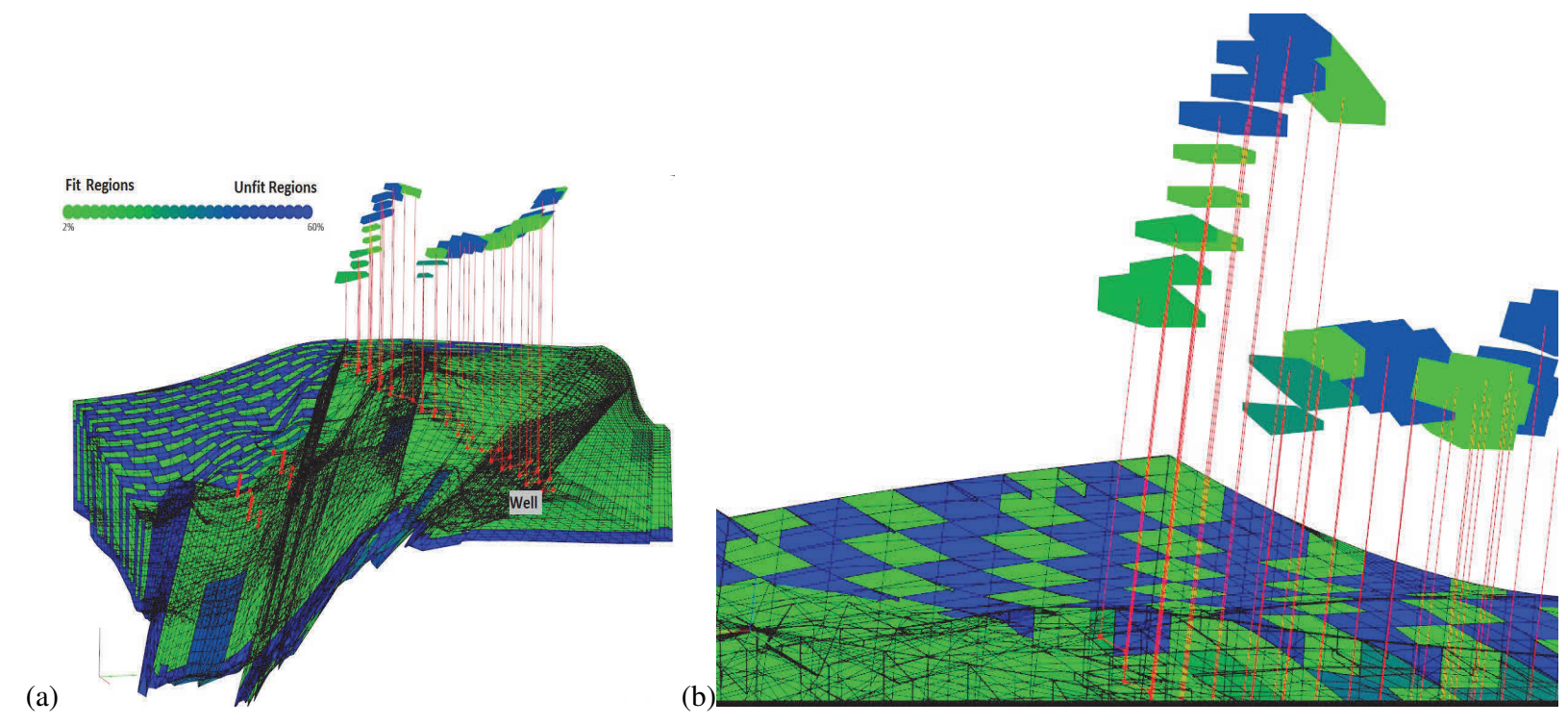

Figure 1: Lollipop-up Visualization

\subsection{Lollipop-up Visualization}

Lollipop-up visualization is an offset technique that provides information about existing well trajectories in the reservoir model (Figure 1). Due to the dense nature of the reservoir models it is hard to achieve a visualization that allows engineers to gain access to the well trajectories without visually removing parts of the reservoir model. Applying partial transparency as an alternative can lead to incorrect appearance of cell color in the case where all the interior layer cells are rendered. It can also lead to loss of depth perception [10]. However, using offsets we can visualize the hidden entities clearly and also maintain the entire reservoir context. Lollipop-up visualization offsets the 3D well perforated cells to a visible height for the engineer to view and learn more about the underground trajectory. It also reflects on the depth of the well point inside the model. The points closest to the surface have shorter offset lengths compared to those deeper down. The connection between the original perforated cell and the offset cell is shown using red lines. Unlike DragMag [25], in this technique we do not alter the offset entity in any way. The offset cell has the same shape, size and color as the original perforated cell within the reservoir model (Figure 1b), thus can provide insights about the underground trajectory cells both in terms of geometry and property values. The visualization also supports mapping the reservoir context to other different geological properties to enable comparing the lollipop-ups with the trend in the remaining cells of the reservoir model. For example, the reservoir model can be mapped to represent water saturation, while the lollipop-ups display the combination of oil pressure and porosity.

\subsection{Single Well Information Circles}

Information circles augment the lollipop-up visualizations by providing numerical information about the geological property mapping within the context of the well trajectory (Figure 2). As seen in Figure 2, the visualization appears at the position of a physical Microsoft Surface tag, thus, can be positioned anywhere on the screen. The circles are arranged in a linear fashion, sorted by size and with equal spaces between each circle. The size of the circle corresponds to the number of well cells with a particular property value (combination of pressure and porisity values). For example, if ten cells correspond to property value $40 \%$ and two cells with $60 \%$, then the circle corresponding to $40 \%$ will be larger. As seen in Figure 2, when a viewer taps on a particular information circle, all the corre-

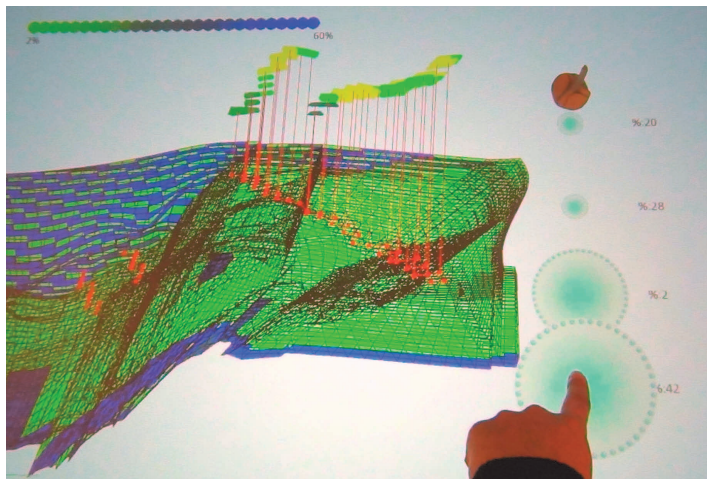

Figure 2: Single well information circles

sponding perforated cells in the lollipop-up visualization get highlighted in yellow, allowing the viewer to identify the offset cells that contribute to the particular information circle. In some situations it may happen that the perforated cells have a wide variety of property values, resulting in large number of circles. Therefore, we allow the circles list to be wrapped and unwrapped. Tapping on a particular circle causes all the circles below to be hidden (wrapping), and on sliding a finger downwards the remaining circles reappear (unwrapping).

\subsection{Multiple Wells Information Circles}

Information circles can also be used to compare multiple wells in the reservoir model. To facilitate this comparison the information circles of each well trajectory are 'hung' from a horizontal pole (Figure 3). Our hanging values metaphor is a twist on the classic histogram: the numbers along the vertical axis at the right represent possible geological property values of perforated cells, calculated using the combination of the two reservoir properties, pressure and porosity (see Section 2.1). Perforated cells that have specific geological property values will be represented as information circles on the hanging graph, with their position along the vertical axis corresponding to their cell value. The radius of each information circle represents the number of cells having the corresponding property value on the vertical axis (similar to the single well information cir- 
cles case, Section 4.2). The information circles are all hanging on the horizontal pole, connected to each other via a spring metaphor. The spring connecting the different information circles will stretch or shorten according to the differences in their property values. This was designed to provide a quick comparison of the different distribution of property values between each of the well trajectories in the reservoir model. Consider the comparison of well A to well E. We can quickly observe from Figure 3 that the property values distribution for well A is more spread out: within the entire well A there are only a small number of cells having property value of $2 \%$ with the remaining majority having property value of $62 \%$. In comparison, well $E$ has cells with a smaller spread of property values (maximum number of cells having property value of $2 \%$, followed by very few cells with value of $12 \%$ etc.), leading to the compressed regions of the spring representing well E. Being able to reflect on the differences in property values distribution between wells can help reservoir engineers to consider economic analysis aspects relating to the well placement. The pink circles at the end of the spring represent the accumulative values of all the information circles along each well trajectory, to help in overall comparison. For example, consider comparing well $\mathrm{C}$ to well $\mathrm{F}$. From the visualization it can be observed that well $\mathrm{F}$ has overall higher accumulative property values (larger pink circle), meaning that more perforated cells are placed in unfit regions (more cells having higher property values/blue regions of the model), while well $\mathrm{C}$ has overall smaller accumulative property values (smaller pink circle), placing it in a relatively better position within the reservoir model (more cells having lower property values/green regions of the model).

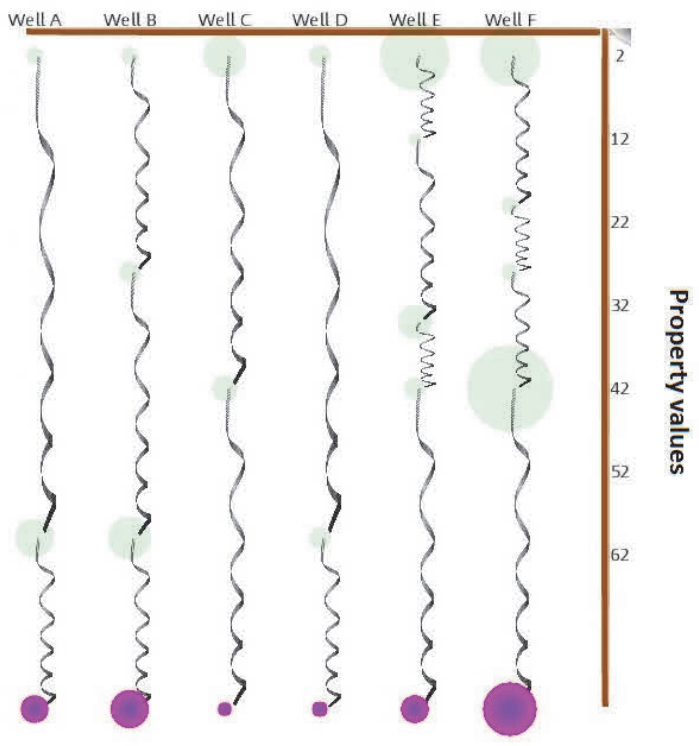

Figure 3: Multiple wells information circles

\subsection{Path Indicator}

Path indicator reflects on exploration of cell specific information done by sketching. This technique allows the engineer to explore the cells of the reservoir model within the context of the model. Due to the need to reach the interior cells of the reservoir model to facilitate sketching in different layers, we need to remove parts of the context. In our current prototype the sketching exploration is limited to orthogonal planes. As can be seen in Figure 4, orthogonal planes can be selected using the walls of the bounding box. Once positioned, the region in front of the plane is removed to allow visibility of sketching. Sketching exploration is augmented with an indicator bubble at the tip of the finger that grows and shrinks in size to indicate growing path navigation through unfit or fit areas. Ideally the indicator bubble should stay small, since this reflects that the path is being placed majorly in fit regions of the reservoir model. The indicator bubble is rendered with partial transparency, to allow the engineer to see the path being sketched. The indicator bubble functions as a short-term memory aid, constantly reflecting the accumulation of the property values to that point by keeping track of the cells that have been passed through in $3 \mathrm{D}$. On removing the finger, the size of the indicator bubble is reset.

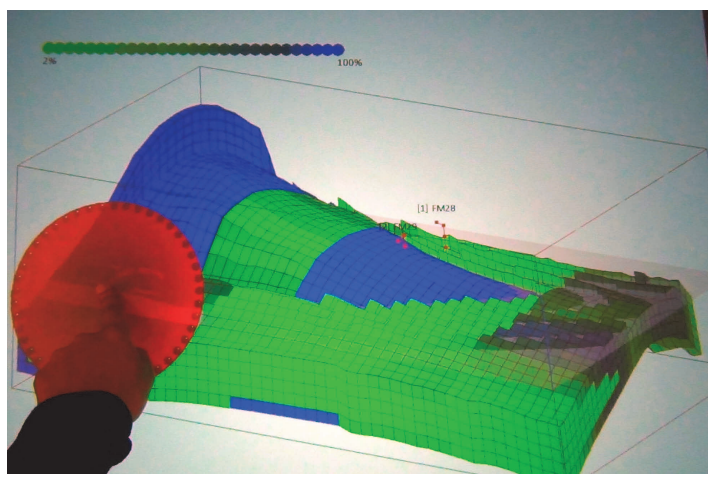

Figure 4: Path Indicator

\section{EXPLORATORY EVALUATION}

We conducted an exploratory evaluation through a set of semistructured qualitative sessions. The goal of these sessions was to better understand the usefulness and the readability of these visualizations. We interviewed three domain experts and three visualization experts. The details of the study are provided in the following sections.

\subsection{Participants}

To learn about the usefulness, benefits, limitations and readability of our visualizations we conducted 6 qualitative semi-structured study sessions. The domain participants were graduate students in the chemical and petroleum engineering department in our university, and had both industry and academic experience with using domain specific software applications. The visualization experts were also graduate student researchers from our university and had research experience in the area of information and scientific visualization.

\subsection{Method}

Each study session was a one-to-one integrated demo, prototype exploration and semi-structured interview, and lasted between 60 to 90 minutes. The sessions started with a brief introduction to the dataset being explored and the visualization techniques being evaluated. As part of pre-study questionnaire, participants were asked about their research background. This was done in order to better understand their exposure and use of visualizations (in general), and their domain expertise. This was followed by a demo of each visualization technique. The participants were encouraged to interact with the visualizations and were asked to think aloud expressing their suggestions, opinions and feedback. During the discussions we asked the participants semi-structured interview questions to encourage their reflection on the usefulness of the techniques, potential advantages and problems of the techniques, and any additional suggestions for improvement. All the sessions were videotaped. Using the video recordings we transcribed the audio for every participant. We performed an open coding of the transcribed data in order to group the discussions (verbal comments) under broad categories and identify interesting observations [20]. We also used the 


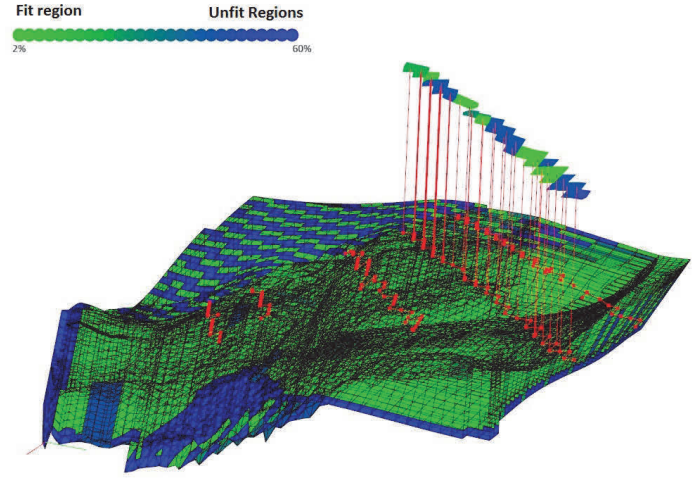

Figure 5: Offset to reflect the same shape as the actual well trajectory.

video recordings to compute the following times: (a) total interaction time - defined as the total of the spans of time the participant was interacting with the visualization either by direct interaction with the visualization or by model manipulation to view the visualization from varying viewpoints; (b) total thinking time - defined as the total of the portions of time when a participant interacted with the visualization without any verbal or physical expression for explaining their thoughts; and (c) discussion time - is the total amount of time that the participant spent discussing the visualization technique.

\section{Results and Discussion}

In the study, 4 out of 6 participants ranked lollipop-up visualization to be most useful of the three visualizations. Two of the visualization experts mentioned liking path indicator better than lollipopup and information circles for exploration. 4 out of 6 participants mentioned that information circles were useful for exploration, but mostly in the case where it was augmented with the lollipop-up visualization. We also observed that although all of our participants liked the visualization techniques, all of them also mentioned limitations and suggestions for improvements for each of the techniques.

From the time recordings we observed that the domain experts spent less time thinking about the lollipop-ups (domain mean rank: 2.67, vis mean rank: 4.33) but almost twice as much time discussing them (domain mean rank: 7.67, vis mean rank: 4.00). Similarly the domain experts spent close to twice as much time on discussing information circles (domain mean rank: 7.33, vis mean rank: 3.67 ). We also observed that when grouped as a whole, the participants had longer interaction time with the lollipop-ups (mean rank: 2.89), than with either the information circles (mean rank: 1.06) or the path indicator (mean rank: 2.06). Similarly, they spend more time discussing the information circles (mean rank: 2.56) compared to lollipop-ups (mean rank: 1.33) and indicator (mean rank: 2.11).

Below we interpret our results to highlight some of the benefits and limitations of our visualizations, and their usefulness to domain experts.

\subsection{Lollipop-up Visualization}

\subsubsection{Benefits and Limitations}

The fundamental benefit of this visualization is that it shows an overview of the hidden well trajectory without compromising the context, "its good for a quick look". Secondly, it was noted that since the model could be mapped to different geological properties it allowed for quick ways to do high level comparison and correlation. Contrary to our current implementation, the shape of the offset well was found to be a limitation of this technique. The majority of our participants mentioned they would prefer to see the same shape of the well being reflected above the surface as shown in Figure 5. "Inversion (of depth) does not help with anything, better to have regular shape (of the well)". Although the depth of the perforated cell is reflected by the height of the trajectory, it introduced discontinuities in the visual. This clashes with the real world modeling of well trajectories and thus was found difficult to interpret. Some of the other limitations of this technique include the overlapping well cells. Although the visualization allows for a quick overview of the geological property distribution in the well trajectory, detailed information is hard to achieve due to the overlap. As seen in Figure $1 \mathrm{~b}$, even though the cells are generally displaced, due to the geometric nature of unstructured corner point cells, there may be some overlap. Interactions that allow each offset cell to be separated and examined individually can remedy such situations, "have a probe, using which you can move over the cells (offset cells) and get more information about them".

\subsubsection{Novelty and Suggestions}

Two of our domain experts mentioned that lollipop-up visualization was visually similar to bubble diagrams, a visualization available in the commercial software Petrel [1]. However, they found the lollipop-up visualization to be novel and useful as it allowed each well perforation cell to be examined. This is in contrast with bubble diagrams which show a single cumulative value for each well trajectory. Another of the domain participant mentioned that in the commercial software, well specific information can be accessed using a layer specific slicing technique. However, slicing is not easy to perform, and hence he preferred lollipop-ups, which provided an overview readily, "In Petrel something of this sort can be done by slicing, but this (lollipop-up visualization) is better than slicing because it is not easy to select a well and go slicing”. All our participants provided suggestions for improving this visualization. Among the many, one interesting suggestion was to use two sets of lollipop-ups in two opposite directions. Rather than collapsing two geological properties into one map, the participant suggested it would be better to have two sets of lollipop-ups each mapped to one property. The participant felt this might improve the ease of comparison and correlation.

\subsection{Information Circles}

\subsubsection{Benefits and Limitations}

One major observed benefit of this technique was the interaction between the information circles and lollipop-up visualization. With the help of this interaction, the location of well cells could be identified; this was specifically useful to identify those cells in the unfit regions requiring consideration. Another benefit of this visualization is that it gives the engineer an idea of the number of cells with a particular property value, and provides insights about where changes have to be made within the collection of well points, ("could be useful in scenarios of economic analysis of wells"). Some limitations of information circles included the initial training time needed to understand the technique. It was initially found to be confusing to interpret for the majority of our participants. One of the domain participant mentioned that since information circles are similar to histograms, it might be better to not introduce a new visual language. Also it was observed that the information circles strength was in interaction with lollipop-ups, "if I knew it (interaction), maybe I could read it (information circles) without your explanation", "now this (interaction) is very useful".

\subsubsection{Novelty and Suggestions}

Although static information circles were not preferred over histograms, the interaction between the lollipop- ups and information circles improved their readability and usefulness. Our domain participants found this to be something new and one mentioned that it 
could be more useful for geologists, "more important for geologists rather than reservoir engineers". One suggestion for this technique was regarding against the use of circles. Alternatively, some participants suggested arranging the perforated cells in clusters to support quick understanding of the visualization.

\subsection{Path Indicator: Benefits and Limitations}

\subsubsection{Benefits and Limitations}

Path indicator was seen as beneficial for domain tasks such as well planning, "useful when designing new wells or to make predictions about your model". Because path indicator allows for somewhat free-form, sketch-based exploration, it allowed the engineer to easily explore the area of importance to them. One obvious limitation of our current protoype is the restricted orthogonal planes for sketching. To support well creation tasks, flexible drawing planes need to be supported.

\subsubsection{Novelty and Suggestions}

All our domain experts mentioned this visualization would be useful within the context of well planning. One of the suggestions to improve this visualization included placing the indicator bubble at an offset. While half of our participants mentioned that they preferred the indicator bubble to be at the tip of the finger for immediate feedback, the other half preferred it to be at an offset, seeing it as a distraction. Two of our visualization experts mentioned that this technique could be useful in collaborative settings, when two or more people could propose different well paths within the model and later compare their findings and strategies.

\subsection{Color Scale}

To facilitate distinct interpretation of the fit and unfit regions, we chose to not use rainbow color scales in this experiment. However, while this worked for some of our participants, "I think it's nice to see the contrast between the blue and green. You can really tell that this is fit and this is unfit", some of the others found it difficult to read very similar shades of green and blue and preferred the use of more distinct colors.

\section{CONCLUSION AND Future Work}

In this paper we described the design and implementation of three visualization techniques created to support the exploration and visualization of well trajectory specific information in 3D reservoir models. We also present a discussion of our findings from two distinct groups of participants (domain experts and visualization experts) who took part in our exploratory study. From these initial set of findings we observed that all the three visualizations have potential benefits and usefulness for the domain. Part of the contribution of our work is blending concepts from scientific visualization and information visualization to come up with techniques that can provide insights about the reservoir models to the domain engineers.

In the near future, we plan to improve our visualizations based on the suggestions provided by our participants. In the long term we would like to improve our sketching techniques, and consider scenarios for creating 3D well configurations intuitively. Using these well trajectory creation scenarios as a task set we would like to conduct another set of task oriented studies to validate the usefulness and intuitiveness of these visualization variations.

\section{ACKNOWLEDGEMENTS}

We would like to thank our colleagues and reviewers for their valuable comments, suggestions and discussions. This research was supported by the Alberta Innovates Academy (AITF) / Foundation CMG Industrial Research Chair in Scalable Reservoir Visualization.

\section{REFERENCES}

[1] Schlumberger. http://www.slb.com/services/software/geo/petrel/simulation.aspx.

[2] tecplotr. http://www.tecplot.com/products/tecplot-chorus/.

[3] J. Bertin. Semiology of Graphics: Diagrams, Networks, Maps. Esri Press, 2011.

[4] S. Bruckner. Interactive illustrative volume visualization. $\mathrm{PhD}$ thesis, $\mathrm{PhD}$ thesis, Vienna University of Technology, 2008.

[5] M. S. T. Carpendale, D. J. Cowperthwaite, and F. D. Fracchia. Extending distortion viewing from 2D to 3D. Computer Graphics and Applications, IEEE, 17(4):42-51, 1997.

[6] M. S. T. Carpendale and C. Montagnese. A framework for unifying presentation space. In Proc. of the 14th annual ACM symposium on User interface software and technology, pages 61-70. ACM, 2001.

[7] L. Castanié, B. Lévy, and F. Bosquet. VolumeExplorer: Roaming Large Volumes to Couple Visualization and Data Processing for Oil and Gas Exploration. In Proc. of the IEEE Visualization (VIS '05), pages $247-254,2005$.

[8] R. Cosse. Basics of reservoir engineering. Technip, 1993.

[9] D. Dopkin and H. James. Trends in visualization for E\&P operations. First Break, 24(3), 2006.

[10] N. Elmqvist and P. Tsigas. A taxonomy of 3D occlusion management for visualization. IEEE Transactions on Visualization and Computer Graphics, 14(5):1095-1109, 2008.

[11] G. W. Furnas. Generalized fisheye views. In Proc. of the SIGCHI conference on Human factors in computing systems (CHI '86), pages $16-23,1986$.

[12] R. Gasteiger, M. Neugebauer, O. Beuing, and B. Preim. The FLOWLENS: A Focus-and-Context Visualization Approach for Exploration of Blood Flow in Cerebral Aneurysms. IEEE Transactions on Visualization and Computer Graphics, 17(12):2183-2192, 2011.

[13] IBM. Open Visualization Data Explorer, OpenDX. http://www.opendx.org/highlights.php?highlight=inaction/oil/, 2002.

[14] O. D. Lampe, C. D. Correa, K.-L. Ma, and H. Hauser. Curve-Centric Volume Reformation for Comparative Visualization. IEEE Transactions on Visualization and Computer Graphics, 15(6):1235-1242, 2009.

[15] S. Y. Lee, K.-W. Lee, and U. Neuman. Interactive visualization of oil reservoir data. Geospatial Visual Analytics, 2008.

[16] D. S. Oliver, L. B. Cunha, and A. C. Reynolds. Markov chain Monte Carlo methods for conditioning a permeability field to pressure data. Mathematical Geology, 29(1):61-91, 1997.

[17] D. K. Ponting. Corner point geometry in reservoir simulation. In $1 s t$ European Conference on the Mathematics of Oil Recovery, 1989.

[18] M. J. Pyrcz and C. V. Deutsch. Geostatistical reservoir modeling. Oxford University Press, 2014.

[19] H. Sonnet, S. Carpendale, and T. Strothotte. Integrating expanding annotations with a 3D explosion probe. In Proc. of the working conference on Advanced visual interfaces, pages 63-70. ACM, 2004.

[20] A. L. Strauss and J. Corbin. Basics of Qualitative Research : Techniques and Procedures for Developing Grounded Theory. SAGE Publications, September 1998.

[21] N. Sultanum, S. Somanath, E. Sharlin, and M. C. Sousa. Point it, Split it, Peel it, View it: techniques for interactive reservoir visualization on tabletops. In Proc. of the ACM International Conference on Interactive Tabletops and Surfaces, pages 192-201. ACM, 2011.

[22] T. Taerum, M. C. Sousa, F. F. Samavati, S. Chan, and J. R. Mitchell. Real-Time Super Resolution Contextual Close-up of Clinical Volumetric Data. In Proc. of the Eurographics/IEEE - VGTC Symposium on Visualization (EuroVis '06), pages 347-354, 2006.

[23] A. Tiwari and W. P. Brown. System and method for simultaneous visualization of fluid flow within well completions and a reservoir, 2011. US Patent App. 13/811,826.

[24] I. Viola, A. Kanitsar, and E. Grller. Importance-Driven Volume Rendering. In Proc. of the conference on Visualization (VIS '04), pages 139-146, 2004.

[25] C. Ware and M. Lewis. The DragMag image magnifier. In Proc. of the conference companion on Human factors in computing systems (CHI '95), pages 407-408, 1995. 